\title{
Not enough time for research? Use of supported funding to promote allied health research activity
}

This article was published in the following Dove Press journal:

Journal of Multidisciplinary Healthcare

\author{
Rachel Wenke ${ }^{1,2}$ \\ Kelly A Weir ${ }^{1-3}$ \\ Christy Noble ${ }^{1,4,5}$ \\ Jill Mahoney' \\ Sharon Mickan ${ }^{1-3}$ \\ 'Allied Health Clinical Governance, \\ Education and Research, Gold Coast \\ Health, QLD, Australia; ${ }^{2}$ School of \\ Allied Health Sciences, ${ }^{3}$ Menzies \\ Health Institute Queensland, ${ }^{4}$ School \\ of Medicine, Griffith University, \\ Southport, QLD, Australia; ${ }^{5}$ School \\ of Pharmacy, The University of \\ Queensland, Brisbane, QLD, Australia
}

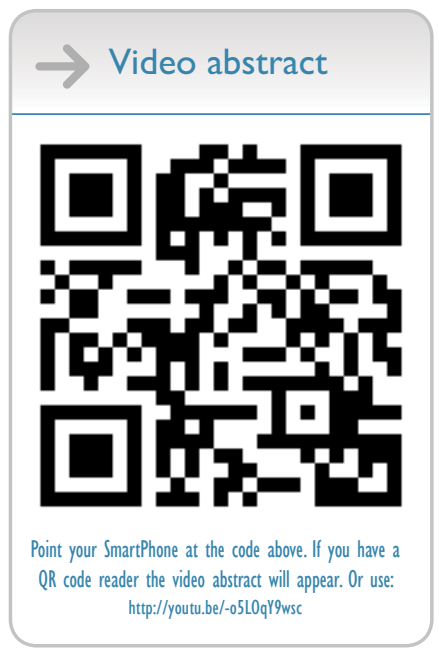

Correspondence: Rachel Wenke Allied Health Outpatients, D block Ground floor, Gold Coast University Hospital, I Hospital Boulevard, Southport, QLD 4216, Australia Tel +6I 75687304 I

Email rachel.wenke@health.qld.gov.au
Purpose: The current project evaluated the impact of a short-term, supported funding initiative that allowed staff from allied health $(\mathrm{AH})$ professions to undertake research activity within rostered employment time. Specifically, the project will report on outcomes pertaining to individual research capacity, research output, and overall satisfaction with the initiative.

Participants and methods: Sixteen AH clinicians $(n=16)$ from six AH professions participated in the evaluation of the initiative, with data being collected within a service improvement framework. Clinicians received up to 4 weeks of protected time relieved from their clinical duties to undertake research activities, including writing for publication, undertaking a systematic review, data analysis, and preparation of ethics applications. An AH Research Fellow provided additional support and mentorship, including the development of an implementation plan. Evaluation included pre-post measures of individual research capacity using a 15-item self-report Research Capacity and Culture (RCC) survey, a post-implementation satisfaction survey, and monitoring of research output achieved.

Results: Statistically significant improvements $(p<0.05)$ were found on 14 out of 15 items on the RCC tool, with meaningful improvements in securing funding, analyzing qualitative data, writing for publication, literature searching skills, and providing advice to less experienced researchers. Overall satisfaction with the initiative was high, with positive comments from $\mathrm{AH}$ professionals (AHPs) regarding the initiative. Research output arising from the initiative included eleven manuscripts being submitted, with six currently in publication and others under review. Conclusion: The preliminary findings support the feasibility of implementing a local, clinical funding model to promote individual research capacity and research output for AHPs. The short-term funding should be supported by local mentorship and guidance. Local barriers and suggestions to optimize implementation, including integrating within existing research infrastructure and using flexible "backfill" options, will also be described.

Keywords: research capacity building, allied health, research engagement, funding

\section{Introduction}

Clinicians working within health care settings are in a pivotal position to identify the most pertinent research questions likely to have the greatest impact on patient care. ${ }^{1}$ It is not surprising then that clinician-driven research may lead to more successful translation of research findings and greater societal impact than academic led research..$^{2-4}$ Allied health $(\mathrm{AH})$ clinicians, which make up the third largest health care workforce (including dietetics, medical imaging, occupational therapy, pharmacy, physiotherapy, psychology, social work, and speech pathology among others), have a prime opportunity to contribute to such research in order to help answer significant 
clinical questions. However, despite clinician motivation and the potential benefits of research participation, ${ }^{5}$ a number of barriers limit an AH professional's (AHP's) ability to undertake research activity.

A recent systematic review revealed that generally, the research culture of AH clinicians within health care has considerable room for improvement and is "associated with a lack of time, skills, resources, team and organisation support and organisational structure". Indeed, "lack of time" has been identified as the most common barrier to AH clinicians participating in research. ${ }^{5-8} \mathrm{AH}$ clinicians working in the public sector frequently prioritize clinical work ahead of research activities because of the lack of clinical "backfill". ${ }^{5,8}$ Clinical backfill in this context refers to the provision of another appropriately qualified clinician to complete usual clinical work, allowing the incumbent to focus on completing their research activity. Due to competing clinical demands, many clinicians wanting to undertake research often do so as unpaid overtime out of work hours or on weekends, a circumstance that is prohibitive for others.

Competitive funding and grant initiatives allowing clinicians to undertake research within work time, while providing clinical backfill to provide their usual clinical services and patient contact time, may address this barrier. Such initiatives are commonly used by public health services and hospital foundations to promote clinician-led research; however, limited evaluation into their impact has been undertaken within AH. ${ }^{9}$ Ried et al $^{10}$ evaluated a small grant funding scheme to support 38 primary health care practitioners to undertake research, including bursaries $(n=24)$, grant writing funds $(n=11)$, and research fellowships $(n=3)$. Grant holders included not only AH clinicians (47\%) but also general practitioners (GPs) (21\%) and other General Practice staff, including nursing professionals (16\%). All clinicians were assigned a designated mentor, who provided expertise and support across the "life" of the research project. Within a 3 -year evaluation period, eleven out of the 34 clinicians published their findings. Improvements in confidence and skills in undertaking research were also reported.

Other studies have reported positive outcomes for clinicians receiving funding bursaries, including research output and improvements in personal and professional development, but the majority of participants were GPs or nursing staff. ${ }^{11,12}$ While funding programs continue across AH health care services to promote clinician research engagement, there is a need to better understand their impact and feasibility to ensure that funds being used are maximizing outcomes.

\section{Local context}

Gold Coast Health $(\mathrm{GCH})$ is the third largest health care organization in Queensland, Australia, and employs a workforce of over $880 \mathrm{AH}$ clinical staff. A recent quality improvement project undertaken within GCH's AH workforce identified "lack of time" as the main barrier to clinicians undertaking research activity, with the clinical demands of their role taking priority. ${ }^{13}$ In addition, the project revealed that AHPs had a low publication ratio compared to other peer health services per capita, and while clinicians had completed data collection for different projects, they indicated that they lacked time during work hours to write-up their findings. To address these needs, the AH workforce and research office proposed a short-term, supportive funding scheme to provide clinicians dedicated time to undertake research activity, while another clinician completed their usual clinical tasks. This scheme was endorsed by GCH's AH leadership group in 2014.

\section{Aim}

The current project aimed to describe the evaluation of a short-term, supported funding initiative that allowed AHPs access to time for research activity within rostered work hours. Specifically, the project evaluated the impact of the initiative on individual clinician research capacity, research output, and overall clinician satisfaction.

\section{Ethics approval and informed consent}

The following project was reviewed by the Gold Coast Hospital and Health Service Human Research Ethics Committee and was deemed a Quality Activity (HREC/16/QGC/326) and was conducted within a service improvement framework. Therefore, informed consent was not sought.

\section{Participants and methods Participants}

A total of $25 \mathrm{AH}$ clinicians were invited to participate in the evaluation. All clinicians were employees of GCH and were successful in receiving funding from the initiative from October 2014 to February 2016.

\section{Funding initiative}

The funding initiative was open to all AHPs within Gold Coast Hospital and Health Service (GCHHS) to receive up to 4 weeks (full-time equivalent) of funding to enable their clinical role to be backfilled with another clinician, so that they could access time to dedicate to a research activity. An initial expression of interest involving a 1-page application 
form was sent out via email to all AHP managers to disseminate to their teams. Research activities eligible for consideration included writing an ethics application, analyzing data, undertaking a systematic review, and writing-up research findings for publication. All applicants required endorsement from their line manager before applying. Applications were independently scored by judges with an $\mathrm{AH}$ background according to predetermined criteria. Successful applicants were then paired with an AH Research Fellow employed within the health service to support clinicians engage in research. The Research Fellow met with the clinician to devise an implementation plan, which then informed how their funded time would be spent by identifying what their key goals and activities would be, any identified risks, and resources required (Supplementary material). During the funded time, the clinician was contacted approximately once a week by the AH Research Fellow to offer any support required and troubleshoot any perceived current or potential barriers. As many of the clinicians were undertaking research projects that already had research teams, mentorship was also received from these existing collaborations.

Four funding rounds of the initiative took place from October 2014 to February 2016, with a median of 17 applicants and five clinicians being funded each round.

\section{Outcome measures}

\section{Individual research capacity}

Clinicians were asked to complete, via Survey Monkey ${ }^{\circledR}$, the Research Capacity and Culture Tool (RCC) survey before and after participating in the funded research period. ${ }^{14}$ This questionnaire uses a 10-point Likert scale across 15 items related to research capacity with a score of 1-3 indicating low success, 4-7 indicating average, and 8-10 indicating above average.

\section{Satisfaction}

After taking part in the funding initiative, clinicians were asked to complete a customized satisfaction and experience survey, also on Survey Monkey ${ }^{\circledR}$. This included five statements that clinicians were asked to rate their level of agreement with on a Likert scale of 1 to 10 . Three open-ended questions were also asked, including how could the funding scheme be improved and an opportunity to provide any other comments or feedback, and whether further research skill development was required.

\section{Research output}

Approximately 12 months following the completion of the funding, the first author, RW, contacted the individual clinicians involved to ascertain what research output had been achieved because of the funding. These outputs were categorized as ethics approval gained, data collection commenced, submitted publication, accepted publication, or published journal article.

\section{Data analyses}

Normality of the data was checked, and paired $t$-tests or Wilcoxon matched pairs tests were undertaken on the RCC data based on distribution of the data (alpha level 0.05). Items that clinicians rated as "unsure" were not included in analyses. Changes were considered meaningful if they moved from within the category of low success to average (ie, from $<3$ to $>4-7$ ) or from average to above average (ie, 4-7 to $>8$ ). Demographic, satisfaction questionnaire data using the Likert scale and research output data were analyzed descriptively. Free-form responses from the satisfaction questionnaire were analyzed by RW qualitatively using content analyses. ${ }^{15}$ Initial comments were reviewed, and then the main ideas were descriptively coded. These descriptive codes were then organized into broader categories and subcategories by the first author (RW) and confirmed by the co-authors.

\section{Results}

As shown in Figure 1, a total of 16 clinicians completed the pre-post evaluation. Four clinicians completed only the pre-evaluation, and four clinicians did not complete any evaluation. Three of the clinicians who did not complete the evaluation used a portion or none of the funding, because of difficulties finding suitable backfill for their clinical role. Clinicians who completed the pre-post evaluation used an average of 17 days (3.4 weeks) of supported research activity time (range 14-20 days) and came from six different professional backgrounds, as shown in Table 1. Most of these clinicians used the funding to either write for publication $(n=6)$, or prepare an ethics application $(n=4)$. Other clinicians used the funding to undertake a systematic review $(n=3)$, perform both data analyses and write for publication $(n=2)$, and collect data $(n=1)$. There was a mixture of both senior and base grade clinicians. Although research experience varied, the majority of clinicians were considered novice researchers, having not undertaken any prior research. Two clinicians were undertaking a $\mathrm{PhD}$ on a project within the health service.

\section{Individual research capacity}

Following participation in the backfill period, clinicians' average total score on the RCC significantly increased by approximately $50 \%$, from a mean score of 62.5 to 100.5 


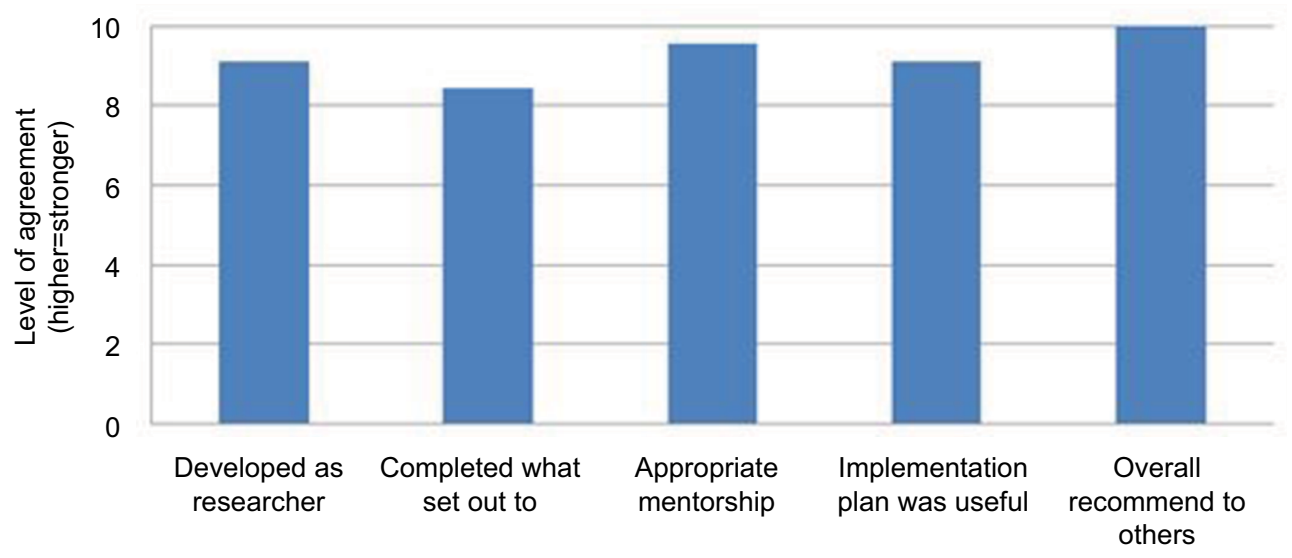

Figure I Clinician satisfaction ratings.

Table I Demographics of clinicians completing evaluation

\begin{tabular}{ll}
\hline Feature & $\mathbf{n}$ \\
\hline Clinical experience & 6 \\
Base grade clinician & 10 \\
Senior clinician & \\
Profession & 4 \\
Speech pathology & 3 \\
Physiotherapy & 3 \\
Dietetics & 2 \\
Occupational therapy & 2 \\
Pharmacy & 2 \\
Psychology & \\
Research activity undertaken & 6 \\
Write for publication & 4 \\
Ethics preparation & 3 \\
Systematic review & 2 \\
Data analyses and write-up & 1 \\
Data collection & \\
\hline
\end{tabular}

out of maximum 150. Fourteen out of 15 items on the RCC demonstrated a statistically significant increase from pre- to post-backfill period (Table 2). The only item that did not show a statistically significant improvement from pre-post was writing a research protocol. Meaningful improvements, whereby clinicians improved from a below average to average range, were found for the items: securing funding, analyzing qualitative data, writing for publication, and providing advice to less experienced researchers. Clinicians also self-reported increased ability in their literature searching skills, moving from the average range to above average range.

\section{Clinician satisfaction}

Clinician satisfaction, from the Likert scale survey items, was overall rated as very high, with participants reporting strong agreement with statements pertaining to the positive experiences of mentoring, their development as a researcher, completing what they set out to achieve, and usefulness of the implementation plan. All participants rated the maximum score (10 out of 10) for agreement of whether they would recommend the scheme to other clinicians.

Qualitative analyses revealed four main themes from the open-ended questions of the questionnaire, as shown in Table 3. These included benefits of the funding initiative, enablers to the initiative, barriers or challenges, and suggestions for improvement. In regards to the benefits of the program, a number of clinicians reported the positive aspect of having the opportunity to be able to dedicate time to undertake research. One clinician commented:

It was a great opportunity, something that is not available in other health districts I have worked for. Most health services have ambitious goals to be involved in QI [quality improvement] or research, but few take the practical step of supporting clinicians with off-line time. [Clinician 14]

Other benefits perceived by clinicians included being able to establish or strengthen networks with other researchers and departments and increased skills and/or confidence in research (Table 3). Regarding enablers, clinicians reported that being backfilled in a part-time capacity or across two or more blocks of time as opposed to one block of full time was helpful:

Going from clinical work to sitting at a computer all day is a big change so it was good to have the break. It also allowed time to get feedback on how the project was progressing. [Clinician 6]

The support received from the research fellows was also greatly appreciated by clinicians: 
Table 2 Individual research capacity and culture survey responses pre- and post-initiative

\begin{tabular}{|c|c|c|c|c|c|c|}
\hline Item on RCC (n) & Pre-mean & SD & Post-mean & SD & $p$-value & $\begin{array}{l}95 \% \mathrm{Cl} \text { for mean } \\
\text { difference }\end{array}$ \\
\hline Finding literature (16) & 6.93 & 1.23 & 8.06 & 1.28 & 0.001 & $-1.70,-0.54$ \\
\hline Critical review of literature (16) & 6.18 & 1.72 & 7.43 & 1.36 & 0.005 & $-1.96,-0.54$ \\
\hline Reference system (eg, Endnote) (16) & 4.50 & 2.39 & 7.18 & 1.93 & 0.001 & $-4.01,-1.35$ \\
\hline Writing research protocol (16) & 5.06 & 2.51 & 6.43 & 2.75 & $\left.0.07\right|^{\wedge}$ & $-2.88,0.13$ \\
\hline Securing funding (15) & 3.93 & 2.73 & 6.20 & 1.69 & $0.002^{\wedge}$ & $-3.56,-0.96$ \\
\hline Writing ethics application (I5) & 4.40 & 2.87 & 6.60 & 2.35 & $0.012^{\wedge}$ & $-3.82,-0.57$ \\
\hline Designing questionnaires (13) & 4.23 & 2.12 & 5.46 & 2.59 & $0.032^{\wedge}$ & $-2.33,-0.12$ \\
\hline Collecting data (14) & 5.31 & 2.57 & 6.93 & 1.48 & 0.014 & $-2.79,-0.40$ \\
\hline Using data management systems (I3) & 4.18 & 2.63 & 6.46 & 2.14 & 0.020 & $-3.78,-0.52$ \\
\hline Analyzing qualitative data (14) & 3.18 & 1.60 & 5.28 & 2.75 & 0.020 & $-3.42,-0.722$ \\
\hline Analyzing quantitative data (I3) & 4.12 & 2.74 & 6.50 & 2.32 & $0.043^{\wedge}$ & -0.34 \\
\hline Writing research report (15) & 4.56 & 2.68 & 6.80 & 1.82 & $0.000^{\wedge}$ & $-2.94,-1.19$ \\
\hline Writing for publication (16) & 3.75 & 2.64 & 6.68 & 1.71 & $0.000^{\wedge}$ & $-3.83,-2.03$ \\
\hline Integrating findings into practice (16) & 6.68 & 2.08 & 7. 25 & 1.61 & 0.046 & $-1.11,-0.01$ \\
\hline Providing advice to less experienced researchers (I5) & 3.73 & 2.21 & 6.67 & 1.75 & $0.000^{\wedge}$ & $-3.85,-2.01$ \\
\hline Total RCC score (16) & 63.43 & 24.60 & 100.50 & 21.40 & 0.000 & $-45.49,-28.63$ \\
\hline
\end{tabular}

Notes: $\wedge$ Paired $t$-test performed. All other tests used Wilcoxon matched pairs.

Abbreviation: RCC, Research Capacity and Culture.

I feel like the luckiest person in the world to have received such great support, not just through having been given the time to dedicate to research, but even more so through the support I have received from [Research Fellow's names here] - many many thanks!!! [Clinician 13]

A barrier reported by clinicians to utilizing the funding period was finding suitable clinicians to backfill their clinical role. Clinicians made several suggestions for improvement to the initiatives, including having more opportunities for the funding, "increased awareness of the scheme" [Clinician 8], and greater detail regarding eligibility criteria. Although not directly related to the initiative, clinicians also made general suggestions for research provision including the use of a designated finance person and statistical support, including access to software.

\section{Research output}

Figure 2 shows the research output of the 16 clinicians who undertook the pre-post evaluation. Of the eleven clinicians who submitted a publication, six have resulted in a publication in a peer-reviewed journal at the time of writing, with a further four clinicians' manuscripts currently under review with a journal. All clinicians who submitted an ethics application had their applications approved, with three commencing data collection. The remaining project has been postponed due to a clinician currently on extended leave. Of the projects that have commenced data collection, one project went on to receive extra, external competitive grant funding.

\section{Discussion}

We aimed to describe the impact of a short-term supported funding initiative to promote research activity in a diverse group of AH clinicians within a single, non-metropolitan health organization. The 16 clinicians participating in the evaluation of the funding initiative reported significantly increased ratings of individual research capacity, with meaningful improvements in literature searching skills, securing funding, analyzing qualitative data, writing for publication, and providing advice to less experienced researchers. Overall satisfaction with the initiative was high, with benefits of the program and suggestions for improvement being reported. Research output included six publications and three new research projects commencing. Findings suggest that the initiative can be feasibly implemented, is well accepted by staff, and can lead to positive improvements in clinician research output and self-efficacy.

The current findings add to the currently limited evidence base describing the outcomes of supported funding initiatives to undertake research in AH. Similar improvements to clinicians' confidence in implementing research have been reported elsewhere following research capacity building interventions, which included funding for $\mathrm{AH}$ clinicians to participate in research activity. ${ }^{10}$ The reported significant improvements in clinician confidence in providing advice to less-experienced researchers may lead to a potential flow-on effect, building research capacity of other individuals within their team. The flow-on effect of clinicians who 
Table 3 Responses to open-ended question of clinician satisfaction survey

\begin{tabular}{|c|c|}
\hline Theme and subtheme & Supporting quote(s) \\
\hline \multicolumn{2}{|l|}{ Benefits of program } \\
\hline Positive opportunity & $\begin{array}{l}\text { [...] there is no way that I could have achieved what I did in the timeframe if I had been doing my clinical role as } \\
\text { well. I feel like my research has significantly progressed thanks to the month of backfill. It had not progressed very } \\
\text { far prior to this! [Clinician 9] } \\
\text { The opportunity to work full time on research (albeit short-lived), provided a huge boost to my research } \\
\text { productivity as I was able to follow questions through to completion in a timely manner. This is often hard when } \\
\text { doing research activity part-time. [Clinician 2] }\end{array}$ \\
\hline Strengthening networks & $\begin{array}{l}\text { I feel like I have established some networks which will also provide ongoing support as I progress with my research } \\
\text { skills. [Clinician 5] } \\
\text { Increased integration within emergency department research to improve local practice and pt care. [Clinician 7] }\end{array}$ \\
\hline $\begin{array}{l}\text { Developed skills or } \\
\text { confidence }\end{array}$ & $\begin{array}{l}\text { I have developed skills in finding evidence, thematic analysis, critical thinking, appraising the literature, using end-note, } \\
\text { teamwork, preparing a manuscript, word processing [formatting]. [Clinician 6] } \\
\text { Increase [in] confidence and ability with literature searching, using software such as CKN, endnote, writing a research } \\
\text { proposal and completing an ethics application. Also, an increased knowledge regarding the steps and systems within } \\
\text { [the health service] to commence and complete a research project. [Clinician 5] }\end{array}$ \\
\hline
\end{tabular}

Enablers to program Using backfill part-time

Appreciated support

Barriers/challenges

Finding backfill
Also, I think the option of having 0.5FTE backfill was good, as it allowed greater flexibility for staff who have roles that are difficult to backfill full-time, also I thought it was useful to have more thinking time, and time to access support, get feedback etc. Full-time research can be very intense especially when you are not conditioned for it! [Clinician I3] Spacing out backfill to be used a few days a week over a longer period of time is very effective. Spacing out research time permits the clinician's research partners time to review projects/documents while the clinician completes clinical work. [Clinician 14]

[Research Fellow's name] was extremely helpful and supportive [...] [Clinician 9]

Thanks [Researchers names here] and team for your efforts into research this year. The funding is a fantastic opportunity and your support is highly appreciated! [Clinician I6]

Long time between notification of funding and backfill time (that was largely due to operational issues). [Clinician 4] Sourcing staff for very short term contracts can be challenging. [Clinician I4]

Suggestions for improvement

More opportunities

Practical research

governance and design

support

More of it! [Clinician 14]

Its not the scheme, but greater staff access to relevant software (such as SPSS) would be useful. [Clinician 2]

The only thing I keep reiterating is that we need a statistician available for GCHHS researchers to help run the correct tests and double-check outputs [...] [Clinician 16]

The other thing which is outside the scope of the funding but would be helpful is a person nominated to assist researchers in their research accounts with collected funding received. [Clinician 16]

Increased awareness of Increased awareness of the scheme. [Clinician 8]

scheme

Suggestions to

It might be useful to include some more detail around eligibility criteria and/or a couple of examples of the types of application projects that were previously successful in obtaining the backfill funding. [Clinician I3]

Abbreviations: GCHHS, Gold Coast Hospital and Health Service; pt, patient; CKN, clinician knowledge network.

have undertaken research activity, being empowered to pass on knowledge and skills to their peers, has been previously reported within $\mathrm{AH} .{ }^{16}$ The effect, consistent with Roger's Diffusion of Innovation Theory, ${ }^{17}$ may explain how, over time, one clinician's research engagement can diffuse through to other clinicians within their team . Building internal capacity and culture within teams is also particularly important for the AH workforce, who are suggested to have a low research culture and skill base. ${ }^{5}$
In addition to reported improved self-efficacy, clinicians produced several research outputs from the initiative. The relatively high success rate of journal article submissions demonstrates the potential benefit of the initiative in helping time-poor clinicians to disseminate their research. Indeed, the present findings revealed a higher publication rate compared to another study evaluating a funding program for AH clinicians (among other health professionals). ${ }^{10}$ The literature has oft-cited the difficulties AHPs have in having their work 


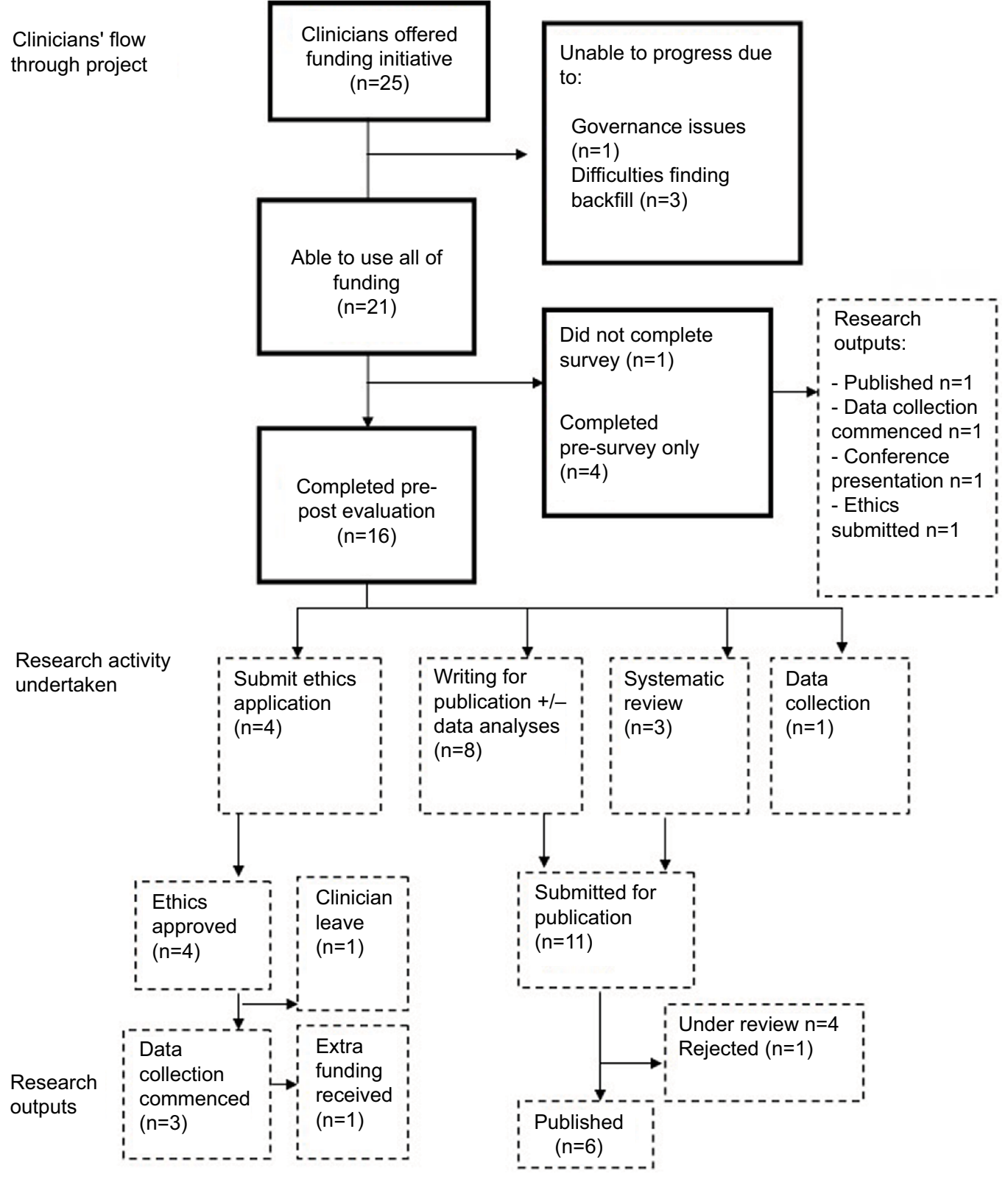

Figure 2 Flow of clinicians through program and research output.

published in peer-reviewed journals. ${ }^{8,18-20}$ The ability to disseminate and contribute to the current evidence base may be of particular value for AHPs, who are reported to have a low research base compared to their medical and nursing peers. ${ }^{6}$ International peer-reviewed journal publications are also a common research performance indicator when comparing research performance of health organizations and therefore may also be important to health service managers.

The use of mentoring from research fellows was reported as a useful facilitator to the success of clinicians achieving their research activity goals as a part of the initiative. The positive benefits of research positions in supporting $\mathrm{AH}$ clinicians to undertake research, including facilitation, has been previously reported in a recent systematic review. ${ }^{21}$ Mentoring and "scaffold" learning from a more experienced researcher can assist in the development of skills that otherwise may have been unattainable for a learner on their own. ${ }^{22}$ Having an implementation plan with clear goals and timeframes set from the outset of the funding period, as well as identification of learning resources including professional development opportunities available, was also valued by clinicians and useful to research fellows in tailoring support to the clinician. Considering that the majority of participants were novice researchers and had not undertaken research before, 
appropriate access to mentorship and learning opportunities was likely a critical component to achieving their research activity goals. ${ }^{23}$

The use of a quality improvement framework to allow for continuous improvement was useful in the implementation of this project, with recommendations for minor adjustments being implemented throughout the four funding rounds. For example, we clarified the eligibility criteria and adjusted the application form to reduce ambiguity around what types of activity would be funded (ie, only activities related to a research project). We also modified the scoring criteria for applications to provide more weighting to the clinical impact of the research activity. While some clinicians reported a lack of statistical support and software and finance assistance (ie, how to operationalize grants) as a barrier to undertaking research, these resources have since been made available within the health service.

\section{Limitations and future directions}

While most clinicians achieved what they had set out to achieve during the funding period, anecdotally other clinicians reported finalizing tasks within their own personal time or using their professional development time within work hours. This was particularly so when clinicians responded to reviewer's comments after submission for publication. Further exploration in how to support clinicians within health care organizations during this publication process may be indicated. Additionally, funds needed to be used within the same financial year. Creating a research trust account to house the funding could allow rollover of funds across financial years, to enable them to be used when appropriate over the "life" of the project/objective, rather than being lost due to end of financial year or used less effectively due to time constraints.

The self-reported improvement in self-efficacy and positive clinician comments about the initiative, although promising, may be influenced by a social bias whereby clinicians wanted to provide a socially desirable response. ${ }^{24}$ While the free-form response in the survey allowed clinicians to share their perceptions, interviews and focus groups may have provided a deeper understanding of clinicians' experiences. Future evaluations of funding initiatives may also wish to use a longer follow-up, and evaluate the impact of the funded research activity on clinical practice and team culture, as these were not evaluated in the present study. Further exploration into specific strategies used by the Research Fellows that may have supported the clinicians may also be indicated.

\section{Recommendations}

The findings suggest that funding initiatives supporting research activity should include access to local mentorship and learning resources to promote accountability and success of the program. While most clinicians were part of an existing research team, having additional research fellow support together with an implementation plan was a useful facilitator to the effectiveness of the initiative. Creative use of funding (including part-time and flexible work arrangements) may also assist clinician productivity during the dedicated research time. Ensuring the initiative is well advertised and that it links-in with existing research infrastructure and supports (ie, statistician and finance officer support) is also recommended. Finally, avenues to support staff in seeking clinical backfill and completing the publication cycle should also be explored. For example, research could be integrated into a clinician's role description or their professional performance plan, so that additional time and resources may be able to be allocated for clinicians to undertake such tasks within their regular working time. Monitoring and feedback, using a rigorous research or service improvement framework locally, may facilitate the outcomes of the funding.

\section{Conclusion}

These findings support the feasibility of implementing a local, clinical backfill funding model to promote individual research capacity and research output for $\mathrm{AH}$ clinicians. Such an initiative may lead to increased dissemination of research and new research projects commencing within $\mathrm{AH}$ professions. The initiative should be implemented within existing research infrastructure with access to local research expertise and resources to support clinicians. In light of the greater societal impact that clinician-led research can have on patient outcomes, initiatives such as this which promote the engagement of clinicians in research activity should be considered as a potential strategy by health organizations.

\section{Data availability}

Data can be made available to readers upon request from Rachel Wenke.

\section{Disclosure}

The authors report no conflicts of interest in this work.

\section{References}

1. Chalmers I, Bracken MB, Djulbegovic B, et al. How to increase value and reduce waste when research priorities are set. Lancet. 2014; 383(9912):156-165.

2. Blevins D, Farmer MS, Edlund C, Sullivan G, Kirchner JE. Collaborative research between clinciians and researchers: a multiple case study of implementation. Implement Sci. 2010;5:76. 
3. Bornmann L. What is the societal impact of research and how can it be assessed? A literature survey. J Am Soc Inf Sci Technol. 2013;64.

4. Cotter JJ, Welleford EA, Vesley-Massey K, Thurston MO. Collaborative community-based research and innovation. Fam Community Health. 2003;26(4):329-337.

5. Borkowski D, McKinstry C, Cotchett M, Williams C, Haines T. Research culture in allied health: a systematic review. Aust J Prim Health. 2016;22(4):294-303.

6. Pager S, Holden L, Golenko X. Motivators, enablers, and barriers to building allied health research capacity. $J$ Multidiscip Healthc. 2012;5:53-59.

7. Lazzarini PA, Geraghty J, Kinnear EM, Butterworth M, Ward D. Research capacity and culture in podiatry: early observations within Queensland Health. J Foot Ankle Res. 2013;6(1):1.

8. Wenke RJ, Mickan S, Bisset L. A cross sectional observational study of research activity of allied health teams: is there a link with self-reported success, motivators and barriers to undertaking research? BMC Health Serv Res. 2017;17(1):114.

9. Hulcombe J, Sturgess J, Souvlis T, Fitzgerald C. An approach to building research capacity for health practitioners in a public health environment: an organisational perspective. Aust Health Rev. 2014;38(3): 252-258.

10. Ried K, Farmer EA, Weston KM. Bursaries, writing grants and fellowships: a strategy to develop research capacity in primary health care. BMC Fam Pract. 2007;8:19.

11. Lee M, Sauders K. Oak trees from acorns? An evaluation of local bursaries in primary care. Prim Health Care Res Dev. 2004;5(2): 93-95.

12. Acton GJ, Yauk S, Hopkins BA, Mayhew PA. Increasing social communication in persons with dementia. Res Theory Nurs Pract. 2007;21(1):32-44.

13. Wenke R. Allied Health: Research Activity and Capacity. Gold Coast Hospital and Health Service: Queensland Health; 2014.
14. Holden L, Pager S, Golenko X, Ware RS. Validation of the research capacity and culture (RCC) tool: measuring RCC at individual, team and organisation levels. Aust J Prim Health. 2012;18(1):62-67.

15. Ryan GW, Bernard HR. Techniques to identify themes. Field Methods. 2003;15(1):85-109.

16. Wenke R, Ward E, Hickman I, et al. Allied health research positions: a qualitative evaluation of their impact. Health Res Policy Syst. 2017;15(1):6.

17. Rogers EM. Diffusion of Innovations. New York: Free Press; 2003.

18. Finch E, Cornwell P, Ward EC, McPhail SM. Factors influencing research engagement: research interest, confidence and experience in an Australian speech-language pathology workforce. BMC Health Serv Res. 2013;13:144.

19. Howard AJ, Ferguson M, Wilkinson P, Campbell KL. Involvement in research activities and factors influencing research capacity among dietitians. J Hum Nutr Diet. 2013;26(Suppl 1):180-187.

20. Boddy J, Daly M, Munch S. The Writing Series Project: a model for supporting social work clinicians in health settings to disseminate practice knowledge. Soc Work Health Care. 2012;51(3):246-270.

21. Wenke R, Mickan S. The role and impact of research positions within health care settings in allied health: a systematic review. BMC Health Serv Res. 2016;16(a):355.

22. Dennen V. Cognitive apprenticeship in educational practice: research on scaffolding, modeling, mentoring and coaching as instructional strategies. In: Jonassen DH, editor. Handbook of Research on Educational Communications and Technology. Mahwah, NJ: Lawrence Erlbaum Associates Publishers; 2004:813-828.

23. Billett S. Readiness and learning in health care education. Clini Teach. 2015;12(6):367-372.

24. Sjostrom O, Holst D. Validity of a questionnaire survey: response patterns in different subgroups and the effect of social desirability. Acta Odontol Scand. 2002;60(3):136-140.
Journal of Multidisciplinary Healthcare

\section{Publish your work in this journal}

The Journal of Multidisciplinary Healthcare is an international, peerreviewed open-access journal that aims to represent and publish research in healthcare areas delivered by practitioners of different disciplines. This includes studies and reviews conducted by multidisciplinary teams as well as research which evaluates the results or conduct of such teams or health

\section{Dovepress}

care processes in general. The journal covers a very wide range of areas and welcomes submissions from practitioners at all levels, from all over the world The manuscript management system is completely online and includes a very quick and fair peer-review system. Visit http://www.dovepress.com/ testimonials.php to read real quotes from published authors. 